\title{
A Specialized Isotope Mass Spectrometer for Noninvasive Diagnostics of Helicobacter pylori Infection in Human Beings
}

\author{
N. M. Blashenkov, E. S. Sheshenya, S. M. Solov'ev, V. D. Sachenko, L. N. Gall, \\ I. V. Zarutskii, and N. R. Gall* \\ Ioffe Physical Technical Institute, Russian Academy of Sciences, St. Petersburg, 194021 Russia \\ St. Petersburg State Polytechnical University, St. Petersburg, 195251 Russia \\ Institute for Analytical Instrumentation, Russian Academy of Sciences, St. Petersburg, 190103 Russia \\ ELMITEK Gmbh, 38678 Clausthal-Zellerfeld, Germany \\ TECHNAN Company, St. Petersburg, 199155 Russia \\ MS-BIO Company, St. Petersburg, 190103 Russia \\ *e-mail: gall@ms.ioffe.ru \\ Received November 18, 2012
}

\begin{abstract}
A specialized isotope mass spectrometer for noninvasive diagnostics of Helicobacter pylori infection in human beings based on the carbon-13 isotope breath test has been designed and constructed. Important stages of the work included (i) calculating a low-aberration mass analyzer, (ii) manufacturing and testing special gas inlet system, and (iii) creating a small-size collector of ions. The proposed instrument ensures ${ }^{13} \mathrm{C} /{ }^{12} \mathrm{C}$ isotopic ratio measurement to within $1.7 \%$ (pro mille) accuracy, which corresponds to requirements for a diagnostic tool. Preliminary medical testing showed that the mass spectrometer is applicable to practical diagnostics. The instrument is also capable of measuring isotopic ratios of other light elements, including $\mathrm{N}$, $\mathrm{O}, \mathrm{B}$ (for $\mathrm{BF}_{2}+$ ions), $\mathrm{Ar}, \mathrm{Cl}$, and $\mathrm{S}$.
\end{abstract}

DOI: $10.1134 / \mathrm{S} 1063785013050040$

The discovery of the fact that infection with Helicobacter pylori bacterium causes gastritis and stomach ulcers in human beings has been marked with the Nobel Prize in physiology or medicine for 2005. Therefore, reliable diagnostics of $H$. pylori infection is an important problem for medicine. Although a number of diagnostic methods are available [1], the isotope breath test recommended by the WHO still remains the "gold standard" since only this test can guarantee the absence of both false positive and negative results, especially for evaluating the efficiency of eradication therapy [2]. In addition, noninvasive diagnostics reduces the risk of hospital-acquired infection and increases the readiness of patients for medical examination.

The isotope breath test consists in administering a small amount of carbamide labeled with stable $\mathrm{C}^{13}$ isotope. In the presence of $H$. pylori bacterium in the stomach, carbamide is decomposed and $\mathrm{C}^{13}$ isotope is absorbed, oxidized to $\mathrm{CO}_{2}$, and eventually eliminated via the lungs. By measuring the excess of $\mathrm{C}^{13}$ in carbon dioxide exhaled by the patient, it is possible to determine the presence of $H$. pylori infection and the degree of stomach mucosa insemination.

To implement this test, an instrument is necessary that must be capable of reliably measuring the $\mathrm{C}^{13} / \mathrm{C}^{12}$ ratio surplus over reference in a range of $1-2 \%$. This possibility can be provided by either specialized isotope mass spectrometers or optical (IR) spectrophotometers, both types having advantages and disadvantages of their own. This Letter describes a TECHNAN MI-150 Helicomass isotope mass spectrometer that is specially intended to carry out the isotope breath test. The instrument has been developed with the cooperation of the Ioffe Physical Technical Institute and the Institute for Analytical Instrumentation (Russian Academy of Sciences, St. Petersburg), TECHNAN Company (St. Petersburg), and MS-BIO Co. (St. Petersburg).

In order to ensure performance of the main function - realization of the isotope breath test - the proposed TECHNAN MI-150 Helicomass isotope mass spectrometer has been designed and constructed with the following analytical characteristics:

Mass resolution

60

Mass range

Isotope sensitivity threshold

$44,45,46$

(no more than)

Diagnostic-significant excess $C^{13}$

concentration over background
$10^{-4}$

$2 \%$ o 
RMS deviation of random component of relative error of isotopic ratio (no more than)

Dimensions $1000 \times 800 \times 400 \mathrm{~mm}$

Weight (with permanent magnet) $60 \mathrm{~kg}$

Power consumption (including PC) $900 \mathrm{~W}$ Mains voltage

AC $220 \mathrm{~V}$

The sum of these analytical characteristics completely ensures realization of the isotope breath test according to WHO standards. Additional requirements in view of the practical application in medicine are (i) a rather short time of analysis and (ii) high reliability of operation (excluding the use of sophisticated, difficult to adjust, and short-working-life units). The instrument design and methodological principles must allow it to be used by staff with a relatively low level of technical training.

The proposed TECHNAN MI-150 Helicomass instrument is a wide-purpose isotope mass spectrometer capable of measuring the isotope composition of light elements (including $\mathrm{H}, \mathrm{Li}, \mathrm{B}, \mathrm{C}, \mathrm{N}, \mathrm{O}, \mathrm{S}$, and $\mathrm{Cl}$ ) [3]. The mass spectrometer is designed as a single-cascade static magnetic device based on a permanent dispersive ring magnet with a radius of $125 \mathrm{~mm}$. A possible alternative variant admits the use of an electric magnet. The analytical stage employs a low-aberration mass analyzer, which was specially calculated for use in this instrument. The mass spectrometer is constructed as a single floor-standing unit with the possibility of separating the analytical stage from electronic circuitry. Passage from one element to another is ensured by using replaceable ion collectors. The instrument is made of fluorine-resistant materials and every possible measure is taken to exclude hydrogen exchange in elements of construction. The electronics has a block structure (which readily admits replacement and modification) and a distributed intelligence, whereby a microprocessor-based online data acquisition system is separated from a PC (running on Windows) and linked to it via a USB port. The mass spectrometer is fully automated and controlled by an original software program package written for Windows, which can be used with both home- and commerciallevel PCs.

The TECHNAN MI-150 Helicomass instrument for medical applications is equipped with an electron-impact ionization source, permanent dispersive $\mathrm{Nd}-\mathrm{Fe}-\mathrm{B}$ magnet, three-collector iondetection system (for 44-, 45-, and 46-amu species), and computer-controlled specialized sample inlet system based on Mamyrin leak valves [4]. Data reset is ensured by a multipass procedure of system purging with compressed air followed by pumping to a forevacuum level, which will be described in a special publication.

High-vacuum pumping in the instrument was ensured by a Pfeiffer Vacuum 071E oil-less pump sys- tem including a $60-\mathrm{L} / \mathrm{s}$ turbomolecular pump and a membrane pump for preliminary evacuation. The residual gas pressure in the instrument prior to sample admission is $10^{-9}$ Torr, and that in the working regime is $10^{-6}$ Torr. The gas inlet system is evacuated by the oil-less membrane pump to a residual gas pressure of about 1 Torr.

The ion source with electron-impact ionization is constructed according to the Nier scheme with horizontal plate electrodes in the region between plates of the exit collimator. The magnetic field was created by external $\mathrm{Nd}-\mathrm{Fe}-\mathrm{B}$ permanent magnets arranged outside the vacuum chamber. The magnetic field strength in the ion source was about $300 \mathrm{G}$. A collector, situated on the ionization chamber side opposite to cathode, takes up to $\sim 95 \%$ of the cathode current. A positive potential of $\sim 30 \mathrm{~V}$ applied to the collector ensures the attraction of electrons, which is necessary to prevent their secondary emission. The cathode represents an yttria-coated U-shaped iridium wire. High-precision isotope measurements are ensured by using a special algorithm of ion source adjustment, which will also be described in a special publication.

The choice of a three-collector ion-detection system is based upon the use of molecular ions of carbon dioxide with a mass number of 44 for the main component $\mathrm{C}^{12} \mathrm{O}^{16} \mathrm{O}^{16}$, a mass number of 45 for the sum of components $\mathrm{C}^{13} \mathrm{O}^{16} \mathrm{O}^{16}$ and $\mathrm{C}^{12} \mathrm{O}^{16} \mathrm{O}^{17}$, and a mass number of 46 for the component $\mathrm{C}^{12} \mathrm{O}^{16} \mathrm{O}^{18}$ with almost insignificant additives of components with two minor isotopes [5]. During medical diagnostics, the two components with mass numbers 44 and 45 are used as bearing information about the $\mathrm{C}^{13} / \mathrm{C}^{12}$ isotopic ratio and the component with a mass number of 46 is used as evidence of the presence of alcohol traces (for rejecting results obtained when this background level exceeds a $2 \%$ o threshold relative to $\mathrm{CO}_{2}$ content). The instrument is operated in a mass-spectrograph regime with simultaneous monitoring of the output signals of three collectors. For tuning and adjustment, the instrument is switched to a mass-spectrometric regime with an accelerating voltage sweep.

The instrument was tested by multiply repeated measurements of the $\mathrm{C}^{13} / \mathrm{C}^{12}$ isotopic ratio in a reference sample, which represented commercial carbon dioxide of medical purity. Simultaneously, additional measurements were performed for the operator expiration. All sequential measurements were carried out using the standard algorithm of intermediate cleaning of the sample inlet system, which was the same as that used during routine medical diagnostics.

Figure 1 presents the results of test measurements. The duration of every measurement (with allowance for the sample-inlet system purging and pumping) was $\sim 7 \mathrm{~min}$; the time required for sequential measurements of a sample and reference was about $15 \mathrm{~min}$. The $\mathrm{C}^{13} / \mathrm{C}^{12}$ isotopic ratio plotted in Fig. 1 was calcu- 


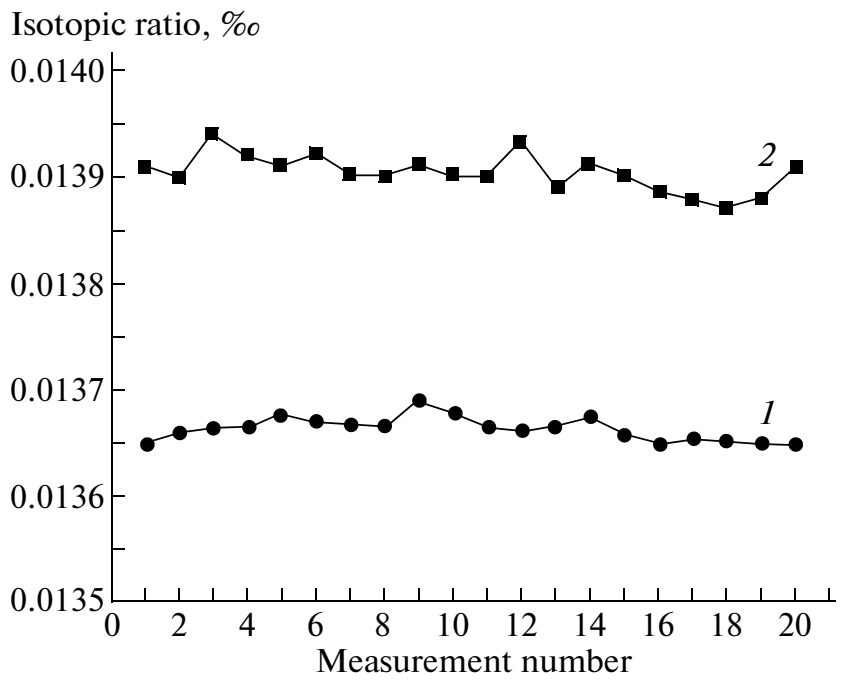

Fig. 1. Variation of the $\mathrm{C}^{13} / \mathrm{C}^{12}$ isotopic ratio in (1) the reference sample and (2) operator Sh exhalation during sequential measurements on a Helicomass instrument with the standard procedure of intermediate purging and pumping of the sample-inlet system (the same as that used during routine medical diagnostics). Every measurement cycle had a 15-min duration.

lated using the conventional formula for isotopic measurements:

$$
R=[I(45) / 33] /[I(44)+I(45) / 33]
$$

where $I(44)$ and $I(45)$ are the signal intensities for mass numbers 44 and 45 , respectively, and 33 is the ratio of resistances in collecting electrodes. In these calculations, the contribution of the minor isotope $\left(\mathrm{O}^{17}\right)$ to the measured value is ignored because the corresponding correction never exceeds $0.5 \%$.

As can be seen, the results of measurements carried out during a working day (for a total of 20 cycles) exhibit a rather insignificant spread, whereby the rootmean-square (RMS) deviation at a confidence probability of $95 \%$ amounts to $\sim 1 / 7 \%$. The RMS deviation calculated for the isotopic ratio in operator exhalation was somewhat greater $(\sim 2.6 \%$ o), which is related to the additional physiological range. It should be noted that systematic differences between the carbon isotope composition of commercial $\mathrm{CO}_{2}$ (of mineral origin) and operator exhalation were reliably detected by the instrument.

Preliminary medical testing of the proposed mass spectrometer was carried out by monitoring $H$. pylori infection in a group of patients using the ${ }^{13} \mathrm{C}$ Carbamide Test preparation, representing a powder of carbamide enriched with ${ }^{13} \mathrm{C}$ isotope to $28-32 \%$. This preparation in a $75-\mathrm{mL}$ dosage form (per single measurement) is produced in Russia and recommended for tests according to the WHO method [6]. For comparison, the same group of patients was diagnosed by using the Breathmat certified commercial mass spectrometer (Finnigan-Mat, Germany) and the same
Surplus isotopic ratio, \%o

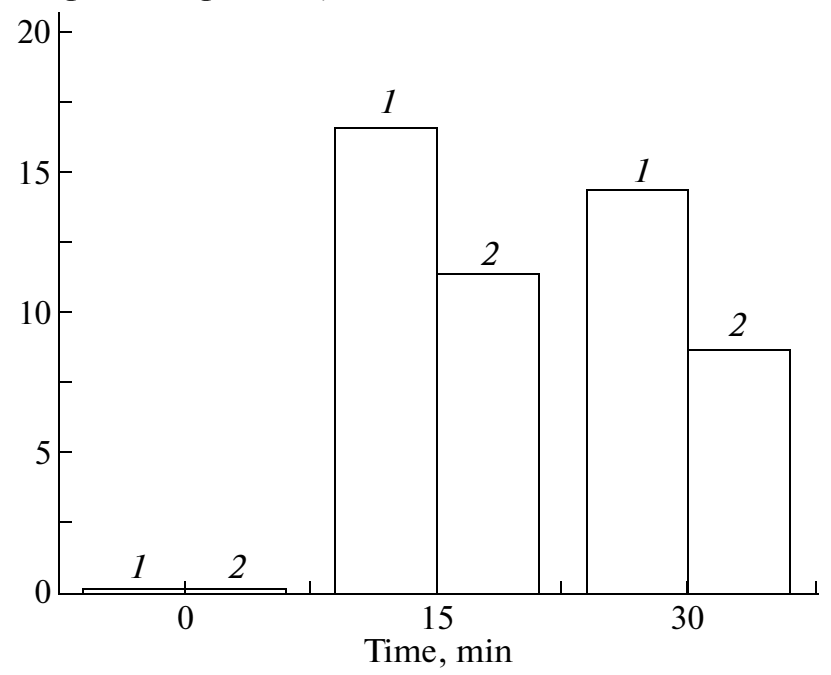

Fig. 2. Temporal variation of the $\mathrm{C}^{13} / \mathrm{C}^{12}$ isotopic ratio in patient $\mathrm{B}$ exhalation measured using (1) Helicomass and (2) Breathmat instruments.

carbamide test preparation. Figure 2 shows typical temporal variation of the $\mathrm{C}^{12} / \mathrm{C}^{13}$ ratio surplus over background measured for one patient by the two instruments. As can be seen, there is good qualitative, and even quantitative, agreement of results (even more so, if we take into account the time shift between measurements). Indeed, the general trends are fully reproduced by both mass spectrometers and the quantitative data differ by no more than $5 \%$ o (i.e., fall within the interval of physiological variance of the human organism).

Medical testing was carried out for a group of seven patients, three of whom had high degree of $H$. pylori insemination, one of whom had a moderate degree of insemination, two of whom had a low degree of insemination, and one of whom showed no evidence of infection. The degree of insemination was estimated using a method recommended by the WHO, according to which the $C^{13} / C^{12}$ ratio surplus over the normal level is determined 15 min after administration of the $\mathrm{C}^{13}$-isotope-rich preparation. The isotopic ratio surplus $\Delta$ was calculated by the following formula:

$$
\Delta=\left[\left(R_{\mathrm{sam}} / R_{\mathrm{st}}\right)-1\right] \times 100 \% \text {, }
$$

where $R_{\mathrm{sam}}$ and $R_{\mathrm{st}}$ are the isotopic ratios in the sample and standard, respectively, as calculated using formula (1).

The analytical characteristics of the TECHNAN MI-150 Helicomass instrument, as determined in the course of testing, show that the mass spectrometer can also be used for measuring the isotopic ratios of other light elements, including $\mathrm{N}, \mathrm{O}, \mathrm{B}$ (for $\mathrm{BF}_{2}+$ ions), $\mathrm{Ar}$, $\mathrm{Cl}$, and $\mathrm{S}$, provided that the instrument is equipped with a special collector system matched with dispersions of the corresponding ion species. 
Thus, the proposed mass spectrometer fully corresponds to the requirements for instruments intended for implementing the isotope breath test and, hence, can be used for medical diagnostics of $H$. pylori infection in human beings. The TECHNAN MI-150 Helicomass instrument is now ready for large-scale tests, which are planned to be carried out with the participation of specialists from Mechnikov Northwestern State Medical University (St. Petersburg).

\section{REFERENCES}

1. L. V. Kudryavtseva, P. L. Shcherbakov, I. O. Ivanikov, and V. M. Govorun, Helicobacter Pylori Infection: Modern Trends in Diagnostic and Therapy (Moscow, 2004) [in Russian].
2. S. I. Rapoport, G. V. Tsodikov, Yu. S. Khodeev, A. M. Zyakun, N. V. Semenova, and N. A. Shubina, Klin. Med., No. 1, 19 (2003).

3. L. N. Gall, A. N. Bazhenov, and A. G. Kuz'min, MassSpektrometriya 5 (4), 295 (2008).

4. B. A. Mamyrin, Prib. Tekh. Exp., No. 1, 205 (1990).

5. A. M. Zyakun, Theoretical Principles of Isotope Mass Spectrometry in Biology (Foton-vek, Pushchino, 2010) [in Russian].

6. G. V. Tsodikov, S. I. Rapoport, and A. M. Zyakun, Ross. Zh. Gastroenterol., Gepatol., Koloproktol., No. 13 (5), 163 (2003).

Translated by P. Pozdeev 